\title{
THE ANGULAR CORRELATION FUNCTION OF FAINT GALAXIES DURING THE DECREASING CORRELATION PERIOD
}

\author{
TONOYA OGAWA \\ Graduate School of Science and Technology, Chiba Univ., Japan \\ BOUDEWIJN F. ROUKEMA \\ Observatoire Astronomique de Strasbourg, France
}

AND

KAZUYUKI YAMASHITA

Information Processing Center, Chiba Univ., Japan

During the period of galaxy formation, many small-length-scale perturbations superimposed on high-amplitude large-length-scale perturbations should have their overdensities boosted into the non-linear regime, while small-scale perturbations in other regions remain linear. The formation of the first galaxies in divclete, high overdensity regions may lead to an initial amplitude of the spatial correlatio iunction of galaxies, $\xi_{0}$, much higher than that expected from linear fluctuation theory. This initially high "bias" would consequently decrease to the near-unity values expected from local observations. Such a Decreasing Correlation Period (DCP) is detected in N-body simulations under certain conditions by several authors; Roukema (1993), Brainerd \& Villumsen (1994), Roukema et al. (1997) and unpublished simulations by one of us (Yamashita).

The DCP is parameterized in a simple way; ending at $z_{t}$. The DCP effects on $w_{0}$, the amplitude of the angular correlation function of faint galaxies, have been estimated and compared with the Hubble Deep Field (HDF) estimate. The DCP is compatible with observation for values of $z_{t}$ in the theoretically expected range $z_{t}>1$ (see Ogawa et al. 1997).

\section{References}

Brainerd, T.G. and Villumsen, J.V. (1994) $A p J, 425,403$

Ogawa, T., Roukema, B.F. and Yamashita, K. (1997) ApJ, 484, 53

Roukema, B.F. (1993) Ph.D. thesis, Australian National Univ., Canberra

Roukema, B.F., Quinn, P.J., Peterson, B.A. and Rocca-Volmerange, B. (1997) MNRAS in press 